\title{
La Gestión Administrativa en la Selección del Talento Humano. Un artículo de revisión de literatura
}

Administrative Management in the Selection of Human Talent. A literature review article

\section{Castro Castillo, Deborah Mercedes}

Maestra en Gestión Pública Universidad César Vallejo, Lima, Perú demeca79@gmail.com, ORCID: 0000-0001-5941-2876

https://scholar.google.com/citations?user=csfDRrUAAAAJ\&hl=es\&authuse $\mathrm{r}=1$

\section{Zapata Quispe, Raquel}

Maestro en Gestión Pública y Gobernabilidad Universidad César Vallejo rzapata24@ucvvirtual.edu.pe raquelzq1@gmail.com, ORCID (0000-00024694-8417), https://scholar.google.com/citations?hl=es\&user=qawF8U0AAAAJ

\section{Díaz Espinoza, Maribel}

Doctora en Administración de la Educación Universidad Tecnológica del Perú, Piura, Perú c20937@utp.edu.pe, ORCID: 0000-0001-5208-8380 https://scholar.google.com/citations?user=uI7T9DUAAAAJ\&hl=es

\section{Reyes Ramírez, Raúl Eduardo}

Maestro en Gestión Pública, Universidad César Vallejo, Lima, Perú, ing.reyesramirez@gmail.com, ORCID: 0000-0002-0993-0568, https://scholar.google.es/citations?hl=es\&user=FW2WIPoAAAAJ

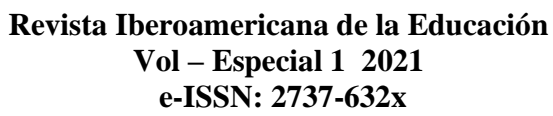

\section{INTRODUCCIÓN}

Leonard (2001) explica que administración es la acción que se realiza para la consecución de algo o la tramitación de un asunto, es acción y efecto de administrar. Es "la capacidad de la institución para definir, alcanzar y evaluar sus propósitos con el adecuado uso de los recursos disponibles". Además, 
menciona que se encarga de coordinar todos los recursos disponibles para conseguir los objetivos empresariales.

En ese sentido, la gestión administrativa tiene relevancia en la gestión de recursos humanos, dado que, la selección de personal es el proceso mediante el cual la compañía contrata talentos idóneos para cubrir puestos, por lo que es muy importante formular estrategias y planes de reclutamiento, pues sin el recurso humano necesario ninguna empresa puede competir.

Según la investigación estadística anual de Glassdoor, el 88\% de los ejecutivos de RR. HH y los expertos de contratación están de acuerdo en que los candidatos bien informados son colaboradores de calidad. Las empresas deben estar abiertas al talento y deben proporcionar a los potenciales postulantes toda la comunicación necesaria para que se interesen en el puesto y se puedan poner en contacto con la empresa.

Así, el 93\% los candidatos opinan que es muy importante estar informados sobre el área de trabajo al que desean postular como su cultura, propósito, valores, planes a futuro. Con esta acción se obtendrá trabajadores eficaces, los que podrán formar parte de programas de desarrollo continúo como producto de un buen proceso de elección del personal.

En Perú, la gran mayoría de empresas no presta la debida atención al procedimiento de selección de recursos humanos, como también no se le da la debida consideración a la sistematización de este proceso.

Aun cuando, en la actualidad no es una prioridad, algunas compañías invierten en el proceso de investigación acerca de estudios sobre 
procedimientos de selección de personal, pues cada organización intenta encontrar el mejor programa para captar al personal idóneo.

Según Herrera, la importancia de estrategias sobre reclutamiento y selección es un proceso vital pues ayudará a concretar la visión y misión de la compañía. El proceso de selección de personal, esta operación está dirigida al campo de los recursos humanos, que tiene la función de ajustar la situación de la vacante. La situación de la vacante está determinada por parámetros y condiciones, estos parámetros y condiciones llevarán a encontrar la persona más idónea para un puesto o un puesto vacante en una empresa. (2017, pg.15).

Por ello, el proceso de reclutamiento y selección responsabiliza una serie de etapas. Estas se inician con la actividad de la demanda de empleo y finalizan cuando se decide contratar a un postulante. Por tanto, se debe tener en cuenta que el reclutamiento es diferente a la selección: el reclutamiento es, sobre todo, divulgación; es decir, dar a conocer que se tienen vacantes; por el contrario, la selección es una tarea de filtrado y está rodeada de elementos condicionales.

Por ello, la gestión del talento es una prioridad para las empresas pues ejecutan la formación, capacitación, selección, la conciliación horaria e incluso se incorporan aspectos de bienestar de los colaboradores haciendo que estos procesos sean mucho más dinámicos y estén integrados con los propósitos generales de la empresa.

Para Martínez, explica que seleccionar el personal forma parte del proceso de provisión del candidato y sucede al reclutamiento; ambos son los periodos de un mismo proceso, la obtención de RR. HH para la compañía. "El 
reclutamiento es una labor de acercamiento, llamada de atención e invitación; la selección es una función de comparación, confrontación, selección y absorción, filtros de entrada y clasificación”. (2019,pg. 226)

Así mismo, en esta etapa de búsqueda del mejor candidato para cubrir la vacante, es donde se han desarrollado diversas opciones de búsqueda de archivos de configuración. Así, puede hacerse mediante la publicación de oportunidades laborales o con una empresa de búsqueda de personal. Uno de las posturas más importantes en esta etapa es la forma en que se difundir el mensaje, es decir, la oferta y los requisitos deben ser precisos. Se puede precisar en la convocatoria el requerimiento de referencias susceptibles de verificación.

De forma complementaria, al elegir al candidato más adecuado, después de un proceso que comprende pruebas técnicas y psicológicas y, en algunos casos, una o más entrevistas se seleccionaran a los postulantes que mejor se adapten a las obligaciones del puesto y a la cultura laboral de la empresa Respecto a la pregunta de investigación se propuso la siguiente interrogante:¿Cuáles son las estrategias de la Gestión Administrativa en la Selección del Talento Humano en las organizaciones?

Así mismo, se propuso como objetivo de la investigación: Identificar las estrategias de la Gestión Administrativa en la Selección del Talento Humano en las organizaciones. 


\section{METODOLOGÍA}

Las revisiones sistemáticas intentan reunir todo el conocimiento de un área específica, destacando lo que se conoce acerca de un tema concreto, a través de los resultados obtenidos en diferentes estudios y ofrecer así recomendaciones para la práctica e investigación futura (Grant\&Booth, 2009; Higgins\& Green, 2011). En la revisión sistemática presentada en este artículo, se analizaron y sintetizaron las evidencias encontradas en investigaciones en torno a la Gestión Administrativa en la Selección del Talento Humano en las organizaciones.

\section{PROCESO DE SELECCIÓN}

Todos los artículos se seleccionaron de las bases de datos académicas tales como: Redalyc, Google académico, Dialnet, Scielo y ScimagoJournal\& Country Rank, utilizando las siguientes palabras clave: gestión administrativa, talento humano, selección de personal, estrategias. Se escogieron estos descriptores, puesto que, son los que interesaban para el estudio.

En este estudio, se tuvieron en cuenta los siguientes criterios de inclusión: artículos publicados en las bases mencionadas líneas arriba entre los años 2010 y 2020, y que estuvieran relacionadas con la gestión administrativa y selección del talento humano.

Los artículos fueron registrados y organizados en un formato, con la finalidad de poder clasificarlas de acuerdo a la base de datos del cual fueron extraídos. El formato almacena información que consideramos relevante para el estudio, los cuales fueron: Temática, año de publicación, título, autor, nombre de la revista científica, idioma y descripción del objetivo. 
Para el análisis de la información recopilada de las bases académicas, se procedió a separar por grupos, es decir, agruparlos por medio de las preguntas claves, algunos eran sobre gestión administrativa, talento humano, selección de personal, estrategias.

\section{ESTRATEGIA DE BÚSQUEDA Y CRITERIOS DE SELECCIÓN}

La Figura 1 da a conocer el proceso de búsqueda, así como los criterios de inclusión/exclusión contemplados. Se utilizó una técnica de exploración bibliográfica haciéndose uso de descriptores (palabras clave) las cuales fueron utilizadas desde las bases de datos académicas, las cuales muestran los criterios de inclusión y exclusión utilizados en el presente trabajo

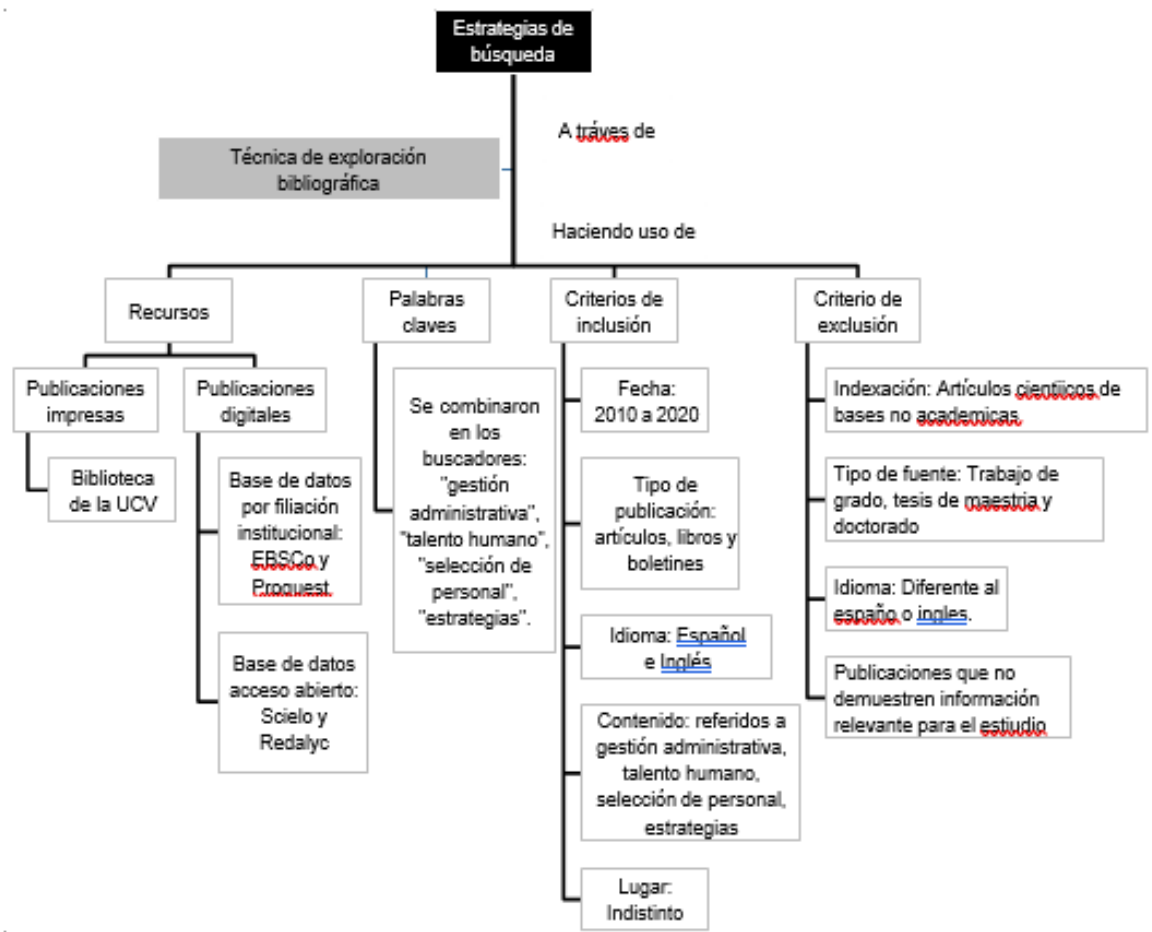

Figura 1: Estrategias de búsqueda empleada 


\section{RESULTADOS}

Resultados de artículos analizados

Se analizaron 40 artículos, de los cuales diez de ellos fueron descartados para el estudio principal. Cabe mencionar, que estos artículos fueron excluidos pues su contenido no concordaba con la información específica de la investigación. En ese sentido, los 30 artículos seleccionados serán descartados pues incluyen las variables de estudio.

Figura $\mathrm{N}^{\circ} 1$

Resultados de artículos analizados

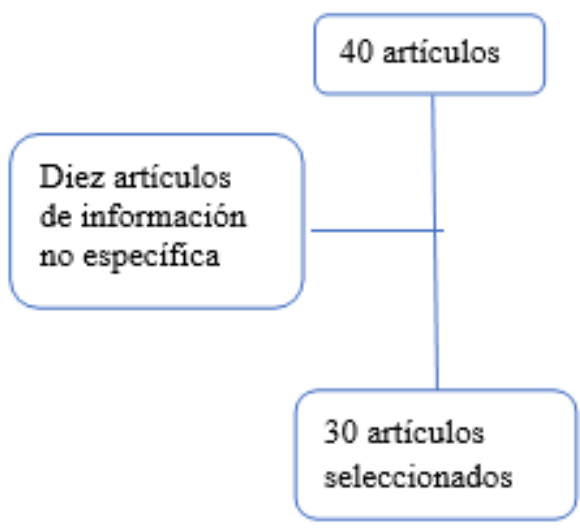

\section{Elaboración: Propia}

De los 30 artículos de las revistas seleccionados, ellos presentan información relacionado la gestión del talento humano, básicamente, a la selección de personal. Además, los artículos son de carácter nacional e internacional, e incluyen en su contenido como procesos, técnicas, métodos y evaluaciones para la captación y selección de los colaboradores. 


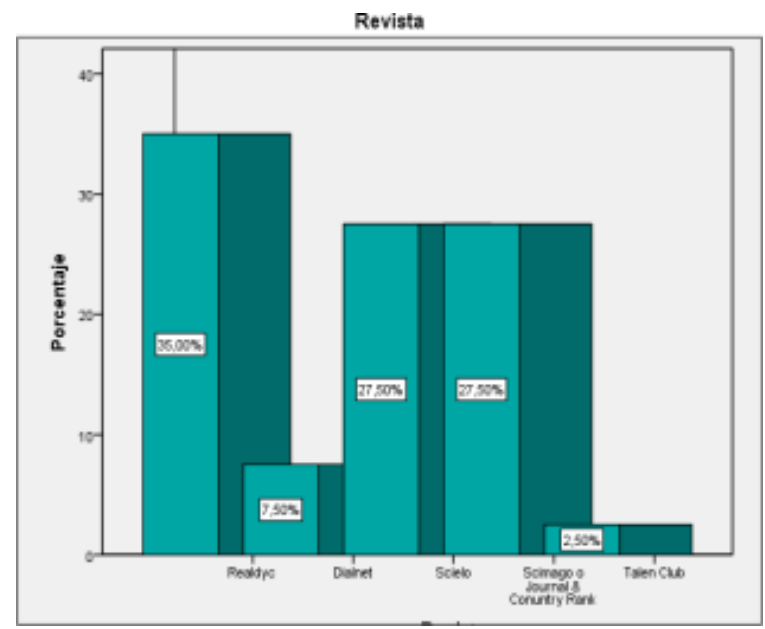

Ilustración 1. Artículos seleccionados Fuente: Elaboración propia

Los 30 artículos seleccionados de acuerdo a las variables son de revistas destacadas donde permite tener información confiable para esta investigación sistemática,dondeel35\%sondeinformacióndelarevistaRedalyc,Dialnet(7,50\% ), Scielo y ScimagoJournal\& Country Rank con27.50\%.

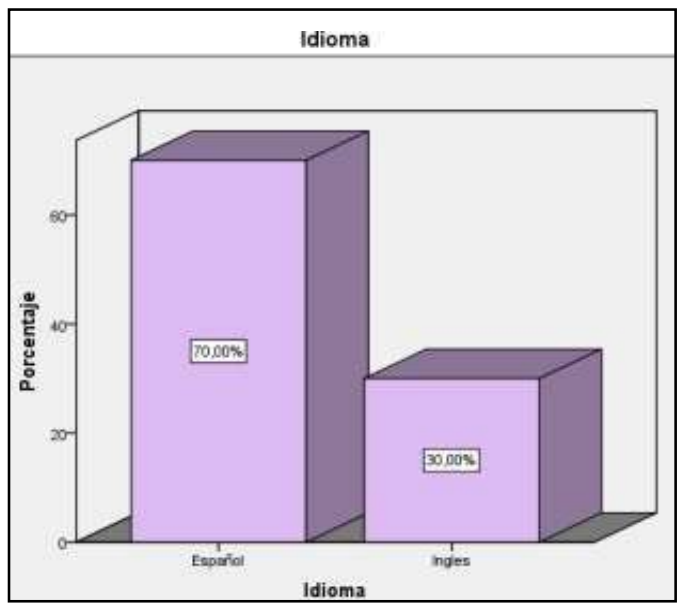

Ilustración 2. Idioma de los artículos Fuente: Elaboración propia 
De acuerdo, al número de revistas seleccionadas para la revisión sistemática el $70 \%$ de las revistas son de idioma español y el 30\% de las revistas son de idioma inglés, así podemos tener diferentes perspectivas para la recolección de información.

\section{REFERENCIA}

Acikgoz, Y. (2019). Employeerecruitment and jobsearch: Towards a multilevelintegration.

ScimagoJournal\& Country Rank. Obtenido de ttps://www.sciencedirect.com/science/article/abs/pii/S1053482218301190 Aguado, D., Rico, R., Rubio, V., \& Fernández, L. (2016). Applicantreactions to social network web use in personnelselection .Scielo. Obtenido de http://scielo.isciii.es/pdf/rpto/v32n3/1576-5962-rpto-32-03-00183.pdf

Article

Aguilera Mancilla, G., NahuatArreguin, J., \& Badillo Morales, O. (2019). Reclutamiento y selección por competencias empresas familiares del Estado de Coahuila. Dialnet. Obtenido de https://dialnet.unirioja.es/servlet/articulo?codigo $=7018150$

Almeda, C. (2018). Las 7 tendencias que están marcando el reclutamiento en 2018 a nivel global.

Obtenido de TalentClue: http://blog.talentclue.com/tendenciasreclutamiento-2018 
Alonso, P., Moscoso, S., \& Cuadrado, D. (2015). Procedimientos de selección de personal en pequeñas y medianas empresas españolas. Redalyc. Obtenido de https://www.redalyc.org/articulo.oa?id=231340289003

Baum, M. (2014). The Effectiveness of RecruitmentAdvertisements and RecruitmentWebsites: Indirect and InteractiveEffects on ApplicantAttraction. ScimagoJournal\& Country Rank. Obtenido de https://onlinelibrary.wiley.com/doi/10.1002/hrm.21571 Bello,M.,Bello,R.,García,M.,\&Casas,G.(2017).Estudioestadísticodelefectod elasimilaridad entre rankings en la selección de personal en un contexto competitivo. Scielo. Obtenido

dehttp://scielo.sld.cu/scielo.php?script=sci_arttext\&pid=S181559362017000300004

Campion,M.,Campion,M.,\&Campion,E.(2019).Usingpracticeemploymentte ststoimproverecruitment and personnelselectionoutcomes for organizations and jobseekers. Psicología aplicada. doi:https://www.apa.org/pubs/journals/features/apl- ap10000401.pdf

Canós, L., Casasús, T., Crespo, E., Lara , T., \& Pérez , J. (2011). Personnelselectionbased on fuzzymethods. Redacly. Obtenido de https://www.redalyc.org/articulo.oa?id=45326927013

Article Castellanos Obregon, J., Osorio Garcia, D., \&TapascoAlzate, O. (2016). Estrategias de movilización,reclutamientoycomposicióndelistaseneleccioneslocales. Scielo. Obtenidodehttp://www.scielo.org.co/scielo.php?pid=S012147052016000200001\&script $=$ sci_abstract\&tlng=es Contreras Pacheco, O. E., Camacho Cantillo, D. C., \&Badrán Lizarazo, R. 
M. (2017). Las Social NetworkingSites (SNS) en los procesos de reclutamiento y selección del talento humano. Revisión de literatura. Redalyc. Obtenido de https://www.redalyc.org/jatsRepo/2654/265452747008/265452747008.pdf Farfán Cabrera, T. (2014). Reclutando profesionales con deseos de empleo. Redalyc. Obtenido de https://www.redalyc.org/pdf/340/34031038004.pdf Fernández Alonso, M., Vásques Padilla, M., Dujarric Bermúdez, G., \& Díaz Simón, N. (2015). Perfil por competencias laborales y modelo de selección de personal para el cargo Técnico A en Gestión de Recursos Humanos. ScimagoJournal\& Country Ran. Obtenido de https://www.researchgate.net/publication/280237480_Perfil_por_competenc ias_laborales_y_modelo_de_seleccion_de_personal_para_el_cargo_Tecnico _A_en_Gestion_de_Recursos_Humanos

Flores Díaz, J. E. (2016). Estrategias para mejorar el proceso de reclutamiento y selección de personal en la Dirección de teleinformática de la gobernación del estado Mérida. Realdyc. Obtenido de https://www.redalyc.org/articulo.oa?id=553057362005

Galarza Iglesias, A. M. (2019). Estrategia de búsqueda de información científica aplicada al reintegro laboral de personas con discapacidad. Scielo. Obtenido de http://www.scielo.org.co/pdf/reus/v22n2/2389-7066-reus-2202-186.pdf

\section{Article}

Galo, N., Ribeiro, P., Mergulhao, R., \& Vidal, J. (2018). Selección de proveedor de servicios logísticos: alineación entre criterios e indicadores. Scielo.

Obtenido de 
http://www.scielo.org.co/scielo.php?script=sci_abstract\&pid=S0121$50512018000300055 \& \operatorname{lng}=\mathrm{en} \& \mathrm{nrm}=\mathrm{iso} \& \operatorname{lng}=\mathrm{es}$

García Castro, P., Gatica Barrientos, M., Cruz Sosa, E., Gatica, K., Vargas Hernández, R., Ramos Velázquez, V., \& Macías Díaz, D. (2016). Procesos de reclutamiento $\mathrm{y}$ las redes sociales. Redalyc. Obtenido de https://www.redalyc.org/pdf/4981/498153966004.pdf

García Izquierdo, A., Derous, E., \&Searle, R. (2015). Reclutamiento and selection in Europe: One stepbeyond. ScimagoJournal\& Country Rank. Obtenido de https://www.elsevier.es/en- revista-revista-psicologia-deltrabajo-organizaciones-370-articulo-recruitment-selection- in-europe-oneS157659621370001X

Gatica Barrientos, M., García Castro , P., Cruz Sosa, E., Luis Gatica, K., Vargas Hernández, R., HernándezGarcía,J.,...

MaciasDíaz,D.(2016).Procesosdereclutamientoylasredes sociales.Redalyc.Obtenidodehttps://www.redalyc.org/pdf/4981/4981539660 04.pdf

Gempp, R. (2014). Coeficiente Phi(Lambda) y la fiabilidad de las decisiones sobre selección de personal.Redalyc.Obtenidodehttps://www.redalyc.org/articulo.oa?id=26432 004003

GulAbbasi, S., ShailTahir, M., \& Abbas, M. (2020). Examining the relationshipbetweenrecruitment\&selectionpractices and businessgrowth: Anexploratorystudy. ScimagoJournal\& Country Rank. Obtenido de https://onlinelibrary.wiley.com/doi/abs/10.1002/pa.2438 
Article Jie, S., \&Vincent, E. (2015). Recruitment and selection in ChineseMNEs.

ttps://www.sciencedirect.com/science/article/abs/pii/S1053482218301190.

Obtenido de

https://www.tandfonline.com/doi/full/10.1080/0958519042000192960

López Gumucio, R. (2010). La selección de personal basada en competencias y su relación con la eficacia organizacional. Redalyc. Obtenido de https://www.redalyc.org/pdf/4259/425941230007.pdf

Lozada Contreras, F., \& Zapata Ramos, M. (2015). What Marketing? A Study on Marketing

Managers' Perception of the Definition of Marketing. ScimagoJournal\&Conuntry Rank. Obtenido de https://pdfs.semanticscholar.org/bf8b/0217b419a999b800a6cb8d1b688fd2f7 4c85.pdf?__ ga=2.191560361.2057460207.1571529860679405240.1571529860

Martínez Reinoso, O. L., \& Vargas Fernández, T. (2019). Procedimiento para la gestión del proceso de reclutamiento y selección del personal en función del desarrollo local. Scielo. Obtenido de http://scielo.sld.cu/scielo.php?script=sci_arttext\&pid=S2310-

\section{$340 X 2019000200225 \&$ lang=es}

Martínez Sánchez, J. A. (2012). EL reclutamiento de personal en el Centro

NAcional de Inteligencia (CNI). Redaly. Obtenido de https://www.redalyc.org/articulo.oa?id=77824828007 
Naranjo Arango, R. (2012). El proceso de selección y contratación del personal en las medianas empresas de la ciudad de Barranquilla (Colombia). Redalyc. Obtenido de https://www.redalyc.org/articulo.oa?id=64623932005

ArticlePérez Redondo, R. (2015). Una relación desequilibrada: la selección de personal en tiempos de crisis. Dialnet. Obtenido de https://dialnet.unirioja.es/servlet/articulo?codigo $=4875442$

Pulido Martínez, H. (2014). De cuestiones internas a problemas internacionales: Acerca de las críticas contemporáneas a los procesos de selección de personal. Redalyc. Obtenido de

https://www.redalyc.org/articulo.oa?id=171032365004

Rahman, M., Aydin, E., Haffar, M., \&Nwagbara, U. (2020). The role of social media in e- recruitmentprocess: empiricalevidencefromdevelopingcountries in social networktheory. ScimagoJournal\& Country Rank. Obtenido de https:/www.emerald.com/insight/content/doi/10.1108/JEIM-12-20190382/full/html

Rodríguez Araneda, M., Navarrete Moraga, R., \&Bargsted Aravena, M. (2017). Problemas éticos y consecuencias reconocidas por psicólogos nóveles en reclutamientoy selección de personal. Scielo. Obtenido de https://scielo.conicyt.cl/scielo.php?script=sci_arttext\&pid=S071869242017000300164\&lang=es

Rueda Contrerar, C., Jiménez Almaguer, K., \& Sánchez Tovar, Y. (2015). Percepciones del impacto de la capacitación, compensación y selección del 
personal en la eficiencia de los proyectos. Scielo. Obtenido de http://www.scielo.org.co/pdf/adter/n27/n27a01.pdf

Ruvalcaba Coyaso, F., \&Vermonden, A. (2015). Lógica difusa para la toma de decisiones y la selección de personal. Redalyc. Obtenido de https://www.redalyc.org/jatsRepo/1872/187243745010/index.html Santarriaga Pineda, M., \& Soto Ramírez, F. (2019). Motivaciones de visita de los miembros de las comunidades de marca virtuales a través de las redes sociales y su relación con la intención de compra, reclutamiento y recomendación boca a boca. Scielo. Obtenido de

Articlehttp://www.scielo.org.bo/scielo.php?script=sci_arttext\&pid=S199437332019000200004

Serna, M. (2018). El papel del diploma escolar en el reclutamiento e internacionalización de las elites manageriales en Uruguay: ¿entre apertura cultural y clausura social. Scielo.

Obtenido de http://www.scielo.org.ar/scielo.php?script=sci_arttext\&pid=S1514$68712018000200243 \&$ lang=es

Valderrama, Y. (2015). Aplicación de la teoría de los subconjuntos borrosos en la gestión de personal. Caso: Valderrama Barrios \& Asociados, Trujillo Venezuela. $\quad$ Redalyc. Obtenido de https://www.redalyc.org/articulo.oa?id=465545899005 Veintimilla Ruiz, J., \& Velásquez Beltran, P. (2017). Modelos de reclutamiento y selección de personal para el área comercial de instituciones 
financieras. Dialnet. Obtenido de

https://dialnet.unirioja.es/servlet/articulo?codigo $=6132035$

Vidal, C., Leopoldo, L., Rojas, J., \& Castro, M. (2017). Desarrollo de Sistema

Web de Reclutamiento y Selección y de Directivos por Competencias mediante PHP CodeIgniter 3.0. Scielo.

Obtenido de

https://scielo.conicyt.cl/scielo.php?script=sci_arttext\&pid=S071807642017000200021\&lang=es

Xianyin Lee, B., \&Wendong, L. (2017). The influencefactors of jobsatisfaction and otrelationship with turnoverintention: Takingearlycareeremployees as anexample. Scielo. Obtenido de http://scielo.isciii.es/pdf/ap/v33n3/psicologia_social1.pdf

Ziegl, S. (2012). Procesos de selección y reclutamiento de las élites: la experiencia reciente de Francia y la apertura del campo de la investigación

Article sobre estos grupos. Entrevista a Agnès van Zanten, por Sandra Ziegler, Propuesta Educativa Número 37 - Año 21 - Jun. 2012 -. Redacly. Obtenido de https://www.redalyc.org/articulo.oa?id=403041708006 